\title{
A Novel Optical Waveguide Microcantilever Sensor for the Detection of Nanomechanical Forces
}

\author{
Kirill Zinoviev, Carlos Dominguez, Jose Antonio Plaza, Víctor Javier Cadarso Busto, and Laura M. Lechuga
}

\begin{abstract}
This study presents a novel generic multipurpose probe based on an array of 20 waveguide channels with microcantilevers acting as optical waveguides operated in the visible range. The principle of operation is based on the sensitivity of energy transfer between two butt-coupled waveguides to their misalignment with respect to each other. The technique can be considered an alternative to the known methods used for the readout of the nanomechanical response of microcantilevers to the external force exerted on them. The cantilever displacement can be detected with a resolution of $18 \mathrm{fm} / \sqrt{\mathrm{Hz}}$. The limit is generally defined by the shot noise of a conventional photodetector used for the readout of the output signal. Real-time parallel monitoring of several channels can be realized. In contrast to devices based on the atomic force microscope detection principle, no preliminary alignment or adjustment, except for light coupling, is required. The detection of the cantilever deflection at subnanometer range was demonstrated experimentally.
\end{abstract}

Index Terms-Beam-propagation method, microcantilevers, microoptoelectromechanical system (MOEMS), optical waveguide, silicon technology.

\section{INTRODUCTION}

$\mathbf{U}$ LTRATHIN microcantilevers produced by standard silicon technology possess low spring constants and allow high sensitivity while combined with a detection system based on the atomic force microscopy (AFM) principle in which a laser beam reflected off the cantilever surface is monitored with a position-sensitive photodetector (PSD) located some distance off the chip. Subangstrom resolution provided by the method allows the detection of changes in deflection or in resonant frequency of the cantilever caused by any kind of reaction occurring on its surface. The principle has recently become widely used in biological research and, in particular, for the readout of the nanomechanical response of microbeams to biospecific interactions produced on one side of the cantilevers that result in the bending of the beams [1], [2].

In general, the systems using PSD work very well. The typical value of sensitivity defined as a fractional change in the detected output signal per unit displacement of the cantilever is about $10^{-3} \mathrm{~nm}^{-1}$ [3]. The typical value of deflection noise

Manuscript received July 28, 2005; revised January 17, 2006. This work was done within the Optonanogen project supported by the EC under Grant IST2001-37239.

K. Zinoviev, C. Dominguez, J. A. Plaza, and V. J. Cadarso Busto are with the Instituto de Microelectronica de Barcelona, Centro Nacional de Microelectronica, Consejo Superior de Investigaciones Científicas, Campus Universidad Autónoma de Barcelona, Barcelona 08193, Spain (e-mail: Kirill. Zinoviev@cnm.es).

L. M. Lechuga is with Instituto de Microelectronica de Madrid, Madrid 28760, Spain.

Digital Object Identifier 10.1109/JLT.2006.872315 density (DND) is in the range of $100-1000 \mathrm{fm} / \sqrt{\mathrm{Hz}}$. To our knowledge, the best noise density obtained in experiments, reported by Fukuma et al. [4], was $17 \mathrm{fm} / \sqrt{\mathrm{Hz}}$. However, to reach this sensitivity, one requires high stability in the functionality of each unit: the source, the sensor, and the detector. Temperature and vibration control are essential in the experiments. Chip replacement normally requires readjustment and realignment. As long as the transducer (cantilever) and the monitor (PSD) are separated in space, the system possesses too many degrees of freedom, which introduces ambiguity in the interpretation of its behavior.

Among the other principles used for the detection of cantilever displacement, the highest sensitivity was demonstrated with optical interferometers [5], where DND was $6 \mathrm{fm} / \sqrt{\mathrm{Hz}}$. However, this principle has not been used in commercially available AFMs [4]. Cantilevers with piezoresistive readouts are less sensitive, with their typical sensitivity being in the order of $10^{-6} \mathrm{~nm}^{-1}$ [6], but have the advantage of permitting integrated devices requiring less adjustment and alignment. Spatial integration of the system, which implies placing all or as many components as possible on one chip, would help to increase the reliability and repeatability of the measurements. The arrays of cantilevers with piezo are compact and frequently facilitate operation in nontransparent liquids [6], [7].

Integrated optical sensor systems are small, relatively easy to produce, sensitive and versatile tools [3], [8]-[10]. Integrated optics sensors with cantilevers have been realized before based on the following: 1) a fiber cantilever used to detect displacements with $600 \mathrm{fm} / \sqrt{\mathrm{Hz}}$ DND [9], 2) optical microcantilevers integrated with multimode interferometer [10], and 3) a conventional GaAs cantilever integrated with a Bragg grating as a photoelastic strain sensor with a sensitivity of $10^{-3} \mathrm{~nm}^{-1}$ [3].

Availability of arrays of the cantilevers allows more versatile and sophisticated experiments to be performed. The probability of results being misinterpreted can be significantly reduced with the presence of reference channels. Several different reactions can be conducted and monitored on one chip [11], [12].

In this paper, we propose an integrated waveguide cantilever sensor composed of an array of 20 independent waveguide channels designed for monitoring biospecific reactions. The sensor can work in static or dynamic modes, either by monitoring the deflection or by monitoring the changes in the resonance frequency of the cantilever. The principle of operation is based on the dependence of coupling efficiency between two buttcoupled waveguides on their misalignment with respect to each other. The advantage of the device is that the transducer is integrated with the receptor on one chip, and the external photodetector is only used for optical power readout. No 




Fig. 1. Schematic view of the sensor.

preliminary alignment or adjustment is needed, except for light coupling into the chip, which does not seriously affect the performance of the device if the coupler is well designed. In this paper, there were two main objectives that we aimed to achieve, namely 1) to prove experimentally that the device can be fabricated with waveguide cantilevers being flat so that their initial deflection is less than $1 \mu \mathrm{m}$ and are initially aligned with static waveguides and 2) to demonstrate that the sensitivity of the device is comparable to the one of the AFM principle-based instruments.

\section{Principle of Operation And Theoretical ANALYSIS OF SENSITIVITY}

The "heart" of the sensor is an optically transparent cantilever beam of submicrometer thickness acting as an optical waveguide. Light from the cantilever is injected into the output waveguide, called the receptor, separated from the cantilever's free end by a short gap $\Delta X$ (see Fig. 1). Both the cantilever and receptor are total internal reflection (TIR) waveguides, and only this type of waveguide is treated in this paper. If the gap $\Delta X$ is in the order of several micrometers, the energy transmitted into the receptor changes dramatically with the displacement of the cantilever's free end in the transversal direction $z$. The idea was to monitor the changes in the power transmitted into the output waveguide to register the deflection of the cantilever.

To study the potential abilities of the device, single-mode operation was analyzed. We modeled the performance for the zero- and first-order waveguide modes separately, considering a freestanding waveguide cantilever able to move in a transversal direction and an output waveguide fixed to a substrate. The structure was assumed to be surrounded by air. Only transverse electric (TE)-polarized modes (electric field is parallel to the $Y$-axis, see Fig. 1) were analyzed. All the simulations were done for $0.7-\mu \mathrm{m}$-thick silicon oxide cantilever (refractive index, 1.46) and $0.12-\mu$ m-thick silicon nitride output waveguide (refractive index, 2.0).

The built cantilevers are wide $(40 \mu \mathrm{m})$ and so are the loworder longitudinal modes, which, after exiting the cantilever, suffer negligible divergence in the horizontal plane (XOY). At the same time, highly confined transversal modes diverge very quickly in the vertical plane (XOZ) so that the energy density becomes inversely proportional to the distance. Using the finitedifference beam-propagation method (FDBPM) [13], [14], we built a two-dimensional (2-D) distribution of the electric field in the vicinity of the cantilever exit and approximated it by the Lorentzian function. Approximation for the zero-order mode was given by the expression

$$
E_{0}(z, x)=\frac{2 A_{1}(x)}{\pi}\left(\frac{A_{2}(x)}{4 z^{2}+\left[A_{2}(x)\right]^{2}}\right)
$$

where $A_{1}(x)=0.6 x+1.24$ and $A_{2}(x)=1.02 x-0.07$. It was assumed that $x=0$ at the cantilever end. The field of the first-order mode was approximated by the two Lorentzian peaks

$$
\begin{aligned}
& E_{1}(z, x)=\frac{2 A_{1}(x)}{\pi} \\
& \quad \times\left(\frac{A_{2}(x)}{4\left(z+A_{3}(x)\right)^{2}+\left[A_{2}(x)\right]^{2}}-\frac{A_{2}(x)}{4\left(z-A_{3}(x)\right)^{2}+\left[A_{2}(x)\right]^{2}}\right)
\end{aligned}
$$

where $A_{1}=2.82+9.64 \exp \left(-0.21(x-4.2)^{2}\right), A_{2}=-0.35+$ $1.87 x-0.08 x^{2}, \quad$ and $\quad A_{3}=-0.167+0.657 x-0.216 x^{2}+$ $0.027 x^{3}$. Both approximations are valid for $x$ containing in the range from 1 to $6 \mu \mathrm{m}$.

The electric field of the fundamental mode of the output waveguide was built on the basis of a solution of Maxwell equations and the propagation constants derived from the dispersion equation [15].

The coupling efficiency was calculated using the overlap integral [16]

$$
\eta(z, \Delta X)=\frac{\left(\int_{-\infty}^{\infty} f(z, \Delta X) g^{*}(z) d z\right)^{2}}{\int_{-\infty}^{\infty} f(z, \Delta X) f^{*}(z) d z \int_{-\infty}^{\infty} g(z) g^{*}(z) d z}
$$

where $f(z, \Delta X)$ is the distribution of the electric field of light exiting the cantilever at the distance $\Delta X$ off the cantilever end, and $g(z)$ is the distribution of the electric field of the fundamental mode of the receptor.

Sensitivity, which is defined as the change in coupling efficiency per unit cantilever displacement, is given by the expression [17]

$$
S=\frac{\partial \eta}{\partial z}
$$

The sensitivity depends both on the gap $\Delta X$ and on the initial displacement of the cantilever, as denoted in Fig. 1 by $z_{0}$. The sensitivity was numerically calculated and plotted in Fig. 2 as a function of $\Delta X$ and $z_{0}$. There is a small asymmetry on the graphs due to nonsymmetrical distribution of the fundamental mode of the output waveguide. The coupling efficiency taken at the points of maximum sensitivity is plotted versus the gap width in Fig. 3. As expected, the coupling efficiency and the sensitivity decrease quickly as the gap $\Delta X$ increases. To achieve high sensitivity with a short gap, the cantilever must be initially displaced to a certain position with precision of several hundred nanometers. Deflection of the cantilever out of this 


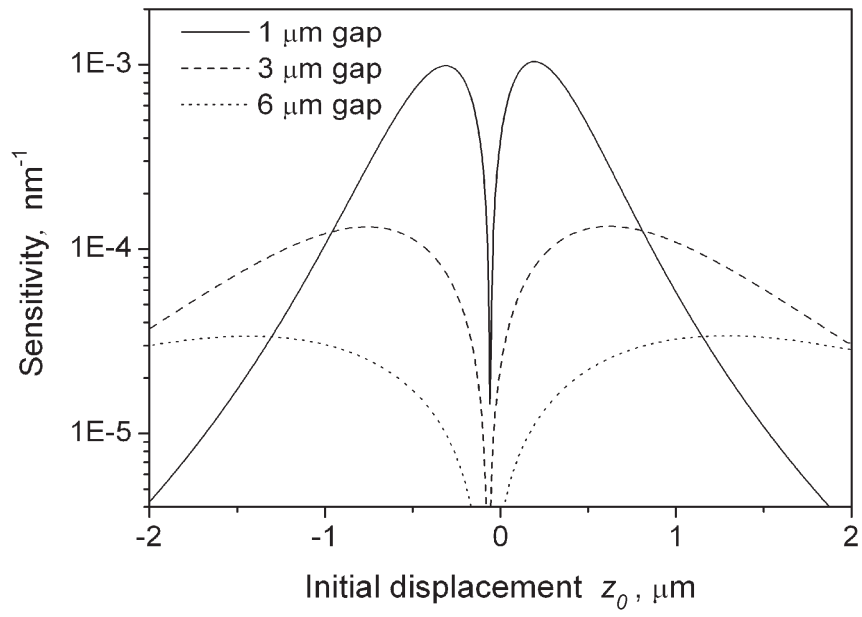

(a)

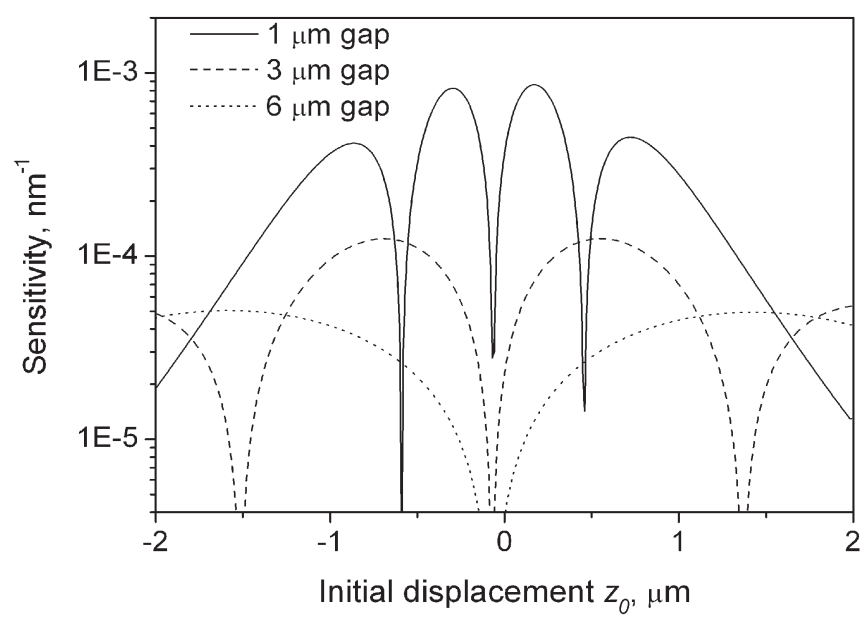

(b)

Fig. 2. Sensitivity $S$ versus initial displacement and the gap width. (a) Zeroorder mode. (b) First-order mode.

range would result in significant decrease in sensitivity. The wider the gap, the lower the precision in the initial deflection is required. The gap width $\Delta X$ is a tradeoff: A short gap allows for high sensitivity and efficiency, whereas a wide gap makes tolerance limits of the initial displacement less strict, which facilitates fabrication of the sensors.

The minimum detectable deflection (MDD) is limited by the shot noise of the photodetector, Johnson noise of the load resistor, the noise in the acquisition system, the cantilever vibration due to the thermal noise, and the noise produced by the laser source. The root-mean-square (rms) shot noise current generated by the photodetector is given by the expression [9]

$$
\begin{aligned}
& \left\langle i_{\mathrm{sn}}\right\rangle=\sqrt{2 e P_{\text {out }} \gamma \Delta f} \\
& \left\langle i_{\text {sn }}\right\rangle=\sqrt{2 e \eta P_{\text {in }} \gamma \Delta f}
\end{aligned}
$$

where $e=1.6 \cdot 10^{-19} \mathrm{C}$ is the electron charge, $\gamma=0.4 \mathrm{~A} / \mathrm{W}$ is the photodetector responsivity, $P_{\text {out }}$ is the optical power of light projected on to the photodetector, $P_{\text {in }}$ is the optical power of light exiting the cantilever, and $\Delta f$ is the spectral bandwidth.

The noise currents of the other origins are either much smaller, like, for example, resistor Johnson noise [17], or dif-



Fig. 3. Coupling efficiency between the cantilever and the output waveguide taken at the points of maximum sensitivity versus the gap width.

ficult to predict because they are dependent on the design of the instrument. Some of noise can be filtered or compensated using differential measurements and lock-in amplification techniques.

The signal-to-noise ratio (SNR) can be found as [9], [17]

$$
\mathrm{SNR}=\frac{\left(\partial i_{\text {out }} / \partial z\right)^{2}}{\left\langle i_{\mathrm{sn}}^{2}\right\rangle}
$$

where $i_{\text {out }}$ is the current generated by the photodetector in response to the output signal.

MDD is defined by an SNR equal to unity [17]. For the given SNR, the MDD can be calculated as

$$
\mathrm{MDD}=\frac{1}{\sqrt{\mathrm{SNR}}}=\sqrt{\frac{2 e \eta \Delta f}{S^{2} P_{\mathrm{in}} \gamma}}
$$

or in terms of DND

$$
\mathrm{DND}=\frac{\mathrm{MDD}}{\sqrt{\Delta f}}=\sqrt{\frac{2 e \eta}{S^{2} P_{\mathrm{in}} \gamma}}
$$

Taking the zero-order mode values of $\eta$ and $S$ corresponding to 3- $\mu \mathrm{m}$ gap between the cantilever and the receptor (Figs. 2 and 3 ) at the position of maximum sensitivity, the expression (8) gives the DND of $80 \mathrm{fm} / \sqrt{\mathrm{Hz}}$ if the power $P_{\text {in }}=1 \mathrm{~mW}$ is supplied to the cantilever. The calculations for the $1-\mu \mathrm{m}$-wide gap give the density of $18 \mathrm{fm} / \sqrt{\mathrm{Hz}}$, which is comparable with the deflection noise densities for the common techniques [4].

\section{DESIGN}

In the embodiment of the built sensor, the cantilever and the output waveguides are located at the same level, although the cantilever's free end and the facet of the output waveguide are misaligned by a fraction of a micrometer. This implies that the cantilever, which is several hundred micrometers long, must be made flat if no other technique for initial biasing correction is applied during the operation of the sensor.

The cantilevers may be fabricated from a single material or as a multilayer structure. The monolayer structure is preferable: It is much easier to find a material that is free of stress 


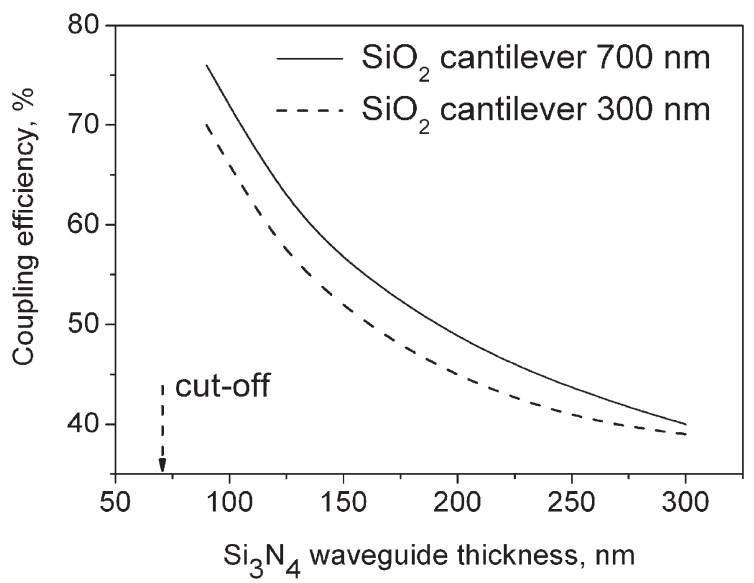

Fig. 4. Coupling efficiency between the input $\mathrm{Si}_{3} \mathrm{~N}_{4}$ waveguide and the $\mathrm{SiO}_{2}$ cantilever versus the IWG thickness.

gradient suitable for the production of straight cantilever beams than to find a combination of two or more different materials resulting in no bimetallic effect that would not bend the beams. Unfortunately, in microelectronics, it is not a trivial problem to find dielectric material free of stress gradient that could be used for waveguide fabrication. Our experiments with low pressure chemical vapor deposition (LPCVD) or plasma enhanced chemical vapor deposition (PECVD) silicon nitrides failed due to the stress gradient in the first one and high absorption losses in the second one. The material we found to fit our requirements was the thermally grown silicon dioxide. The film is perfectly transparent and demonstrates low stress gradient if the bottom layer of a few hundred nanometers is eliminated.

As the refractive index of the silicon dioxide is low and the film is grown on silicon substrate, it cannot form a TIR waveguide over the substrate, unlike the $\mathrm{SiO}_{2}$ cantilever in air. Therefore, over the substrate, light was delivered to the cantilever along a silicon nitride waveguide, called the input waveguide (IWG). At the anchoring area, the IWG being deposited over the silica buffer forms a junction with the cantilever beam, and the latter is an extension of the buffer (see Fig. 1). Generally speaking, nearly $100 \%$ of light can be coupled from $\mathrm{Si}_{3} \mathrm{~N}_{4}$ to the cantilever if the IWG was tapered at the junction. If no taper is provided, light partially radiates in air at the point where the IWG ends. However, relatively low radiation loss can be achieved without taper because a big part of the fundamental mode of a thin $\mathrm{Si}_{3} \mathrm{~N}_{4}$ waveguide is concentrated in the buffer. The efficiency of the coupling, which is calculated using the 2-D FDBPM [13], depends on the thickness of the $\mathrm{Si}_{3} \mathrm{~N}_{4}$ layer and may reach $75 \%$ value when the thickness approaches the 70-nm value corresponding to the cutoff condition of the fundamental mode (see Fig. 4). Coupling efficiency increases with decreasing $\mathrm{Si}_{3} \mathrm{~N}_{4}$ thickness because the mode drops further into the $\mathrm{SiO}_{2}$ layer. Production of a taper would require additional technological efforts, which were omitted in this work where the objective was to prove the concept of the device, so light coupling into the cantilever was realized via the evanescent field of the fundamental mode of the IWG.

The electric field of the transversal fundamental mode of the IWG is located off the cantilever symmetry axis, whereas

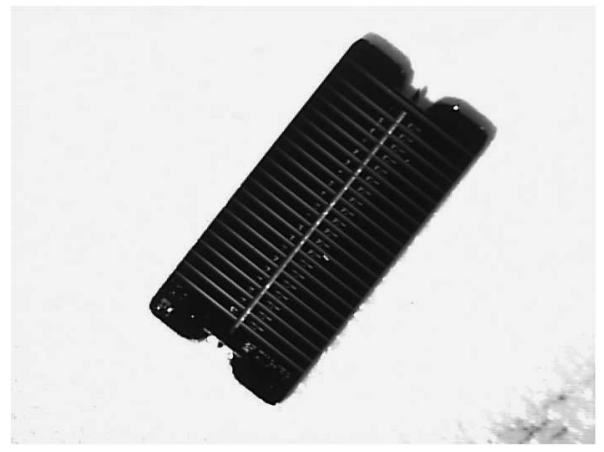

(a)

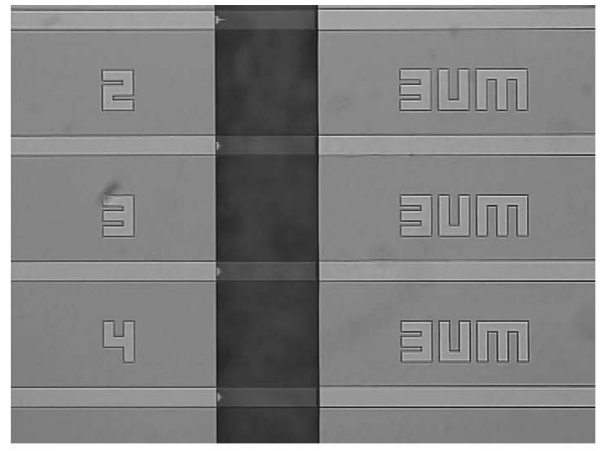

(b)

Fig. 5. (a) Photograph of the fabricated chip. (b) Magnified photo of the cantilevers.

the waveguide cantilever is a symmetrical structure with the modes distributed symmetrically. This results in light from the IWG coupling in all the modes existing in the cantilever. Multimode operation is actually a problem: Orthogonality of the modes implies their independent propagation with different velocities, depending on the parameters of the cantilever, and leads to an unpredictable distribution of light intensity both in transversal and longitudinal directions due to small variations in the cantilever parameters. Therefore, multimode operation is best avoided either by fabricating a single-mode cantilever or by filtering the unwanted modes in some way.

Light coupling into the IWG can be realized either by directly focusing onto the waveguide facet or by means of a diffraction grating coupler implemented on top of the waveguide. The latter is the most convenient and, if the coupler is well designed, is a more effective method, as it does not require the fine alignment of direct focusing.

\section{FABRICATED DEVICE}

The fabricated device presented in the photograph in Fig. 5 contains an array of 20 waveguide channels. Samples with $200-\mu \mathrm{m}$-long cantilever beams were produced. The cantilevers were $500 \mathrm{~nm}$ thick and, in air, supported two guiding modes, according to the simulations. Silicon nitride input and output waveguides were $120 \mathrm{~nm}$ thick. The cantilevers on chip were located in a common cavity, which is a reach-through hole located in the center. Both the cantilevers and the waveguides were $40 \mu \mathrm{m}$ wide. The external facets of the input and output waveguides were made at the very edge of the chip. This helped 




Fig. 6. Profiles of the cantilevers obtained with a confocal microscope. Inset: Scanning electron microscopy (SEM) images of the cantilevers.

to avoid waveguide facet polishing and to provide relatively efficient coupling using direct focusing into the chip and to collect light exiting the receptor using the full numerical aperture.

\section{Characterization}

A chip with $200-\mu \mathrm{m}$-long cantilevers and 3- $\mu \mathrm{m}$-wide gaps was tested. The profiles of the cantilevers measured with a confocal microscope are presented in Fig. 6. The cantilevers are practically flat, so that the cantilever end and the output waveguide facet are misaligned by a few hundred nanometers.

Fig. 7 shows a schematic view of the experimental setup used to measure the amplitude of modulation of the output signal induced by vibration of the cantilever at the resonance frequency. The chip was located on a piezoelectric actuator (Piezomechanik PST150/10x10/18) connected to a sine waveform synthesizer. Light from an He-Ne laser $(632.8 \mathrm{~nm}$, $7.5 \mathrm{~mW}$ ) was coupled into the chip using direct focusing with an objective lens $(40 \times$, numerical aperture (NA) 0.65$)$ and was collected upon exiting by another objective $(40 \times$, NA 0.65) before being directed to a silicon photodetector (PD, Hamamatsu S1337-33BR) connected to an oscilloscope and an acquisition system for spectrum analysis through a low-noise amplifier with bandwidth of $5-45 \mathrm{kHz}$ at full-width at halfmaximum (FWHM). Light from the same laser source after splitting was focused by a lens with a focal distance of $75 \mathrm{~mm}$ on the cantilever near its free end. The reflected beam was projected onto a two-sectional PSD to monitor the displacement of the cantilever. Assuming the reflected beam has Gaussian profile (distortion due to the cantilever bending was neglected) given by

$$
P(x, y)=\frac{2 P_{0}}{\pi \sigma^{2}} \exp \left(-2 \frac{x^{2}+y^{2}}{\sigma^{2}}\right)
$$

the difference in voltage generated by the PSD sections can be represented by the expression

$$
\Delta V=\frac{1}{2} P_{0}\left[\operatorname{erf}\left(\sqrt{2} \frac{a-d}{\sigma}\right)+\operatorname{erf}\left(\sqrt{2} \frac{a+d}{\sigma}\right)\right] \mathrm{Rs} \cdot R_{\mathrm{PSD}}
$$

where $P_{0}$ is the total intensity of the beam illuminating PSD, $\sigma=L \Theta_{\max }$ is the size of the beam, $L$ is the distance between the cantilever and the PSD, $\Theta_{\max }$ is the divergence angle of the reflected beam defined by the focusing lens, $d$ is half of the gap width between the photodetector sections, Rs is the responsivity of the PSD, $R_{\mathrm{PSD}}$ is the load resistors in the PSD circuitry, erf is the error function, and $a=\left(3 \Delta Z / L_{\text {cant }}\right) L$ is the displacement of the spot at the PSD defined by the cantilever length $L_{\text {cant }}$ and by the cantilever displacement $\Delta Z$. Cantilever profile corresponding to the fundamental resonance mode and expressed by the formula $z=\kappa x^{3 / 2}$ was considered.

Coupling efficiency provided by direct focusing into the IWG was about $5 \%$. According to the measurements, about $50 \%$ of that was coupled from the IWG into the cantilever. Thus, the losses were about $-16 \mathrm{~dB}$ after the light reached the cantilever. The power of light exiting the output waveguide and measured in direct current $(\mathrm{dc})$ mode was $0.015 \mathrm{~mW}(-27 \mathrm{~dB}$ with respect to the laser power), which corresponded to the 60$\mathrm{mV}$ voltage generated by the photodetector on the load resistor $\left(R_{L}=10 \mathrm{k} \Omega\right)$. The noise amplitude registered in the experiment was $0.05 \mathrm{mV}$. The rms shot noise voltage density generated by the photodetector was calculated as $\left\langle i_{\mathrm{sn}}\right\rangle R_{L}=1.4$. $10^{-8} \mathrm{~V} / \sqrt{\mathrm{Hz}}$. Working in the bandwidth of $40 \mathrm{kHz}$, the noise produced by the photodetector would be $2.8 \mu \mathrm{V}$, which is almost 20 times less than that experimentally observed. The reason was the mechanical vibration of the setup and no filtering of laser noise. The spectrum of the output signal when no voltage was applied to the piezo actuator is presented in Fig. 8. There is a clear resonance behavior near $13 \mathrm{kHz}$ with a $Q$-factor of 12 . When the excitation voltage of variable frequency and amplitude was applied to the piezo actuator, the output signal did not show any oscillations when the cantilever was out of the resonance. In resonance mode, the photodetector demonstrated periodical change in the photocurrent at a frequency of $13.1 \mathrm{kHz}$. The modulation amplitude of both the output voltage and the cantilever displacement linearly increased with the amplitude of voltage supplied to the piezo actuator (see Fig. 9). The change in the output voltage per unit cantilever displacement was $15 \mu \mathrm{V} / \mathrm{nm}$. The initial displacement of the cantilevers, defined by the fabrication, was about $0.4 \mu \mathrm{m}$ upward. Looking at Fig. 9 , it can be concluded that the behavior of the coupling efficiency curve near this point is quite linear.

Working in the spectral range of $1 \mathrm{~Hz}$ with the noise only generated by the photodetector, it would be possible to detect the cantilever displacement with a precision of $\pm(1.4$. $\left.10^{-8} \mathrm{~V} / \sqrt{\mathrm{Hz}}\right) /\left(15 \cdot 10^{-6} \mathrm{~V} / \mathrm{nm}\right)=0.93 \cdot 10^{-3} \mathrm{~nm} / \sqrt{\mathrm{Hz}}$.

The spectrum of the cantilever vibration when exciting the cantilever by the piezo actuator is presented in Fig. 10. The excitation voltage at $13.1 \mathrm{kHz}$ applied to the piezo actuator was $50 \mathrm{mV}$ and corresponded to the cantilever oscillation amplitude of about $1.7 \mathrm{~nm}$. It is worth mentioning that some modulation of the output signal was due to coupling efficiency modulation resulting from the misalignment of the chip and the objective lens 1. In the inset in Fig. 10, the spectrum of the output signal is presented. The driven voltage of the piezo actuator was $50 \mathrm{mV}$ at $11.1 \mathrm{kHz}$, and frequency was shifted out of the cantilever resonance. The amplitude of the output modulation signal was comparable to the amplitude of the modulation 




Fig. 7. Schematic view of the experimental setup.

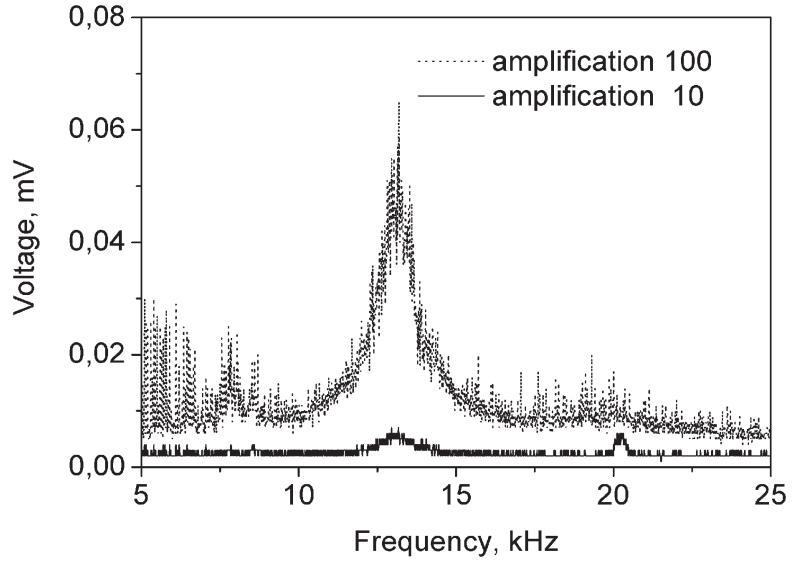

Fig. 8. Spectrum of the output signal. The peak at $13.1 \mathrm{kHz}$ corresponds to the cantilever vibration at resonance frequency induced by thermal noise and external vibration. The other peaks on the graph are due to the proper noise of the acquisition system and the noise induced by modulation of light coupling due to mechanical vibration of the setup. The spectra were taken with amplification factors of 10 and 100 .



Fig. 9. Experimentally measured displacement of the cantilever and modulation amplitude of the output signal versus the amplitude of alternating current (ac) voltage with frequency of $13.1 \mathrm{kHz}$ applied to the piezo actuator. The cantilever was in mechanical resonance mode.

caused by cantilever vibration due to the temperature. Going back to the graph where resonant excitation of the cantilever

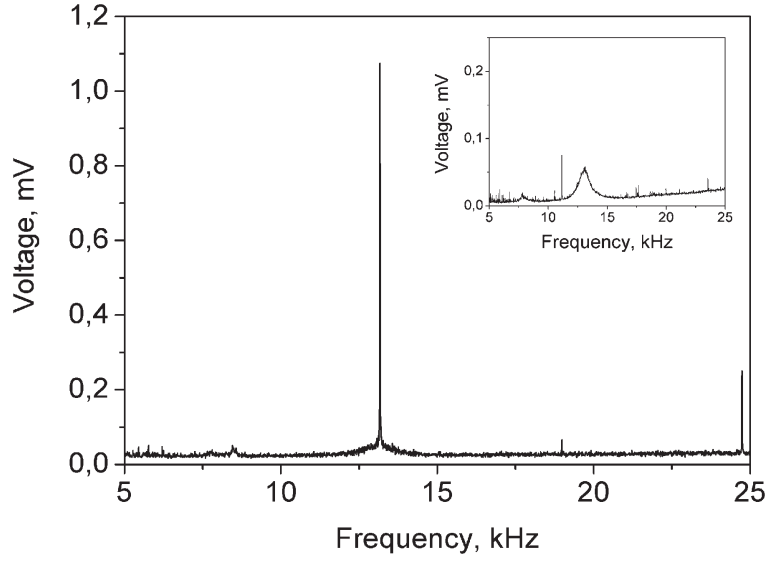

Fig. 10. Spectrum of the output signal. The peak at $13.1 \mathrm{kHz}$ corresponds to the cantilever vibration at resonance frequency induced by piezoelectric actuator. The excitation voltage of the piezo actuator was $50 \mathrm{mV}$. The cantilever oscillation amplitude was about $1.7 \mathrm{~nm}$ at this frequency. Inset: Spectrum of the output signal taken after the actuation frequency was shifted to $11.1 \mathrm{kHz}$.

is shown, we can tell that the SNR was about 40 (voltage amplitude at resonance over the voltage of the resonance). This makes it possible to detect cantilever displacement with a precision of approximately $0.04 \mathrm{~nm}$ with the setup and data acquisition system used in the experiment.

\section{CONCLUSION}

A novel multichannel integrated optical sensor based on butt-coupled waveguide microcantilevers is presented. The theoretical analysis and experiments demonstrated that the device possesses sufficient sensitivity for the detection of subangstrom-scale cantilever deflections. The sensitivity of the technique is comparable to one using the AFM detection principle but is more attractive for applications where the parallel real-time monitoring of several specific reactions on one chip is needed. The device does not need preliminary adjustment or alignment except for light coupling, and it can work in static or dynamic modes, either by monitoring the deflection or by monitoring changes in the resonance frequency of the cantilever. 


\section{ACKNOWLEDGMENT}

The authors thank J. Tamayo and M. Álvarez for help in preparing the experiments.

\section{REFERENCES}

[1] R. McKendry, J. Zhang, Y. Arntz, T. Strunz, M. Hegner, H. P. Lang, M. K. Baller, U. Certa, E. Meyer, H.-J. Güntherodt, and C. Gerber, "Multiple label free biodetection and quantitative DNA-binding assays on a nanomechanical cantilever array," Proc. Natl. Acad. Sci. US, vol. 99, no. 15, pp. 9783-9788, Jul. 2002.

[2] J. Tamayo, A. D. L. Humphris, A. M. Malloy, and M. J. Miles, "Chemical sensors and biosensors in liquid environment based on microcantilevers with amplified quality factor," Ultramicroscopy, vol. 86, no. 1/2, pp. 167-173, Jan. 2001.

[3] C. Kocabas and A. Aydinli, "Design and analysis of an integrated optical sensor for scanning force microscopies," IEEE Sens. J., vol. 5, no. 3, pp. 411-418, Jun. 2005.

[4] T. Fukuma, M. Kimura, K. Kobayashi, K. Matsushige, and H. Yamada, "Development of low noise cantilever deflection sensor for multienvironment frequency-modulation atomic force microscopy," Rev. Sci. Instrum., vol. 76, no. 5, p. 053704, May 2005.

[5] C. Schonenberger and S. F. Alvarado, "A differential interferometer for force microscopy," Rev. Sci. Instrum., vol. 60, no. 10, pp. 3131-3134, Oct. 1989.

[6] J. Thaysen, A. Boisen, O. Hansen, and S. Bouwstra, "Atomic force microscopy probe with piezoresistive read-out and a highly symmetrical Wheatstone bridge arrangement," Sens. Actuators A, Phys., vol. 83, no. $1-3$, pp. 47-53, May 2000.

[7] P. A. Rasmussen, J. Thaysen, S. Bouwstra, and A. Boisen, "Modular design of AFM probe with spattered silicon tip," Sens. Actuators A, Phys., vol. 92, no. 1-3, pp. 96-101, Aug. 2001.

[8] J. van Lith, P. V. Lambeck, H. J. W. M. Hoekstra, R. G. Heideman, and R. R. Wijn, "The segmented waveguide sensor: Principle and experiments," J. Lightw. Technol., vol. 23, no. 1, pp. 355-363, Jan. 2005.

[9] R. Budakian and S. J. Putterman, "Force detection using a fiber-optic cantilever," Appl. Phys. Lett., vol. 81, no. 11, pp. 2100-2102, Sep. 2002.

[10] E. Ollier, P. Philippe, C. Chabrol, and P. Mottier, "Micro-opto-mechanical vibration sensor integrated on silicon," J. Lightw. Technol., vol. 17, no. 1, pp. 26-29, Jan. 1999.

[11] M. Alvarez and J. Tamayo, "Optical sequential readout of microcantilever arrays for biological detection," Sens. Actuators B, Chem., vol. 106, no. 2, pp. 687-690, May 2005.

[12] M. Yue, H. Lin, D. E. Dedrick, S. Satyanarayana, A. Majumdar, A. S. Bedekar, J. W. Jenkins, and S. Sundaram, "A 2-D microcantilever array for multiplexed biomolecular analysis," J. Microelectromech. Syst., vol. 13, no. 2, pp. 290-299, Apr. 2004.

[13] P. C. Lee, D. Schulz, and E. Voges, "Three-dimensional finite difference beam propagation algorithms for photonic devices," J. Lightw. Technol., vol. 10, no. 12, pp. 1832-1838, Dec. 1992.

[14] D. Yevick and B. Hermansson, "Efficient beam propagation techniques," IEEE J. Quantum Electron., vol. 26, no. 1, pp. 109-112, Jan. 1990.

[15] E. Anemogiannis and E. N. Glytsis, "Multilayer waveguides: Efficient numerical analysis of general structures," J. Lightw. Technol., vol. 10, no. 10, pp. 1344-1351, Oct. 1992.

[16] R. G. Hunsperger, Integrated Optics: Theory and Technology. Berlin, Germany: Springer-Verlag, 1984.

[17] G. G. Yaralioglu, A. Atatar, S. R. Manalis, and C. F. Quate, "Analysis and design of an interdigital cantilever as a displacement sensor," J. Appl. Phys., vol. 83, no. 12, pp. 7405-7415, Jun. 1998.

Kirill Zinoviev received the Master's degree in radio engineering from Vladimir State University, Vladimir, Russia, in 1994 and the Ph.D. degree in engineering from Moscow State Academy of Engineering and Computer Science, Moscow, Russia, in 1997.

He is currently with the Group of Chemical Transducers, National Centre of Microelectronics, Barcelona, Spain. His research interest is on the design, fabrication, and characterization of the sensors based on integrated optics and fabricated using silicon technologies.
Carlos Dominguez received the M.S. and Ph.D. degrees in chemistry from the Universidad Computense de Madrid, Madrid, Spain, in 1980 and 1985, respectively.

He became a member of the scientific staff at the Institute of Microelectronics of Barcelona (Centro Nacional de Microelectronica, Consejo Superior de Investigaciones Científicas), Barcelona, Spain, in 1986. Since 1991, he has been a Senior Scientific Researcher. He is involved in materials and process development for new transducers and sensors. Currently, he is working on the development of an integrated optical technology based on silicon for chemical and biochemical sensors and broadband telecommunications applications.

Jose Antonio Plaza was born in Cerdanyola del Vallés, Barcelona, Spain, in 1968. He received the physicist degree and the Ph.D. degree in electronics engineering from the Autonomous University of Barcelona, in 1992 and 1997, respectively.

Currently, he is working in the Department of Silicon Technologies and Microsystems, National Centre of Microelectronics (CNM), Bellaterra, Spain. $\mathrm{He}$ has been focused on technology development, design and characterization of microsystems such as accelerometers, pressure sensors, and gas sensors, and techniques such as anodic bonding. Now, he has joined the nanotechnology group of the CNM, and his research is focused on the fabrication of microelectromechanical systems/nanoelectromechanical systems for nanotechnology applications.

Víctor Javier Cadarso Busto was born in Sabadell, Spain, on October 17, 1981. He received the degree in physics from the Universidad Autónoma de Barcelona, Barcelona, Spain, in 2003.

In 2003, he joined the Chemical Transducers Group at the Instituto de Microelectrónica de Barcelona (Centro Nacional de Microelectronica, Consejo Superior de Investigaciones Científicas). His fields of interest are integrated optics on silicon substrates for broadband telecommunications and optical sensors.

Laura M. Lechuga received the Ph.D. degree in chemistry from the University Complutense of Madrid, Madrid, Spain, in 1992.

She was the Head of the Biosensors Group at the Microelectronics National Center (Consejo Superior de Investigaciones Científicas), Madrid. Her main research areas are in the technological development of integrated optical and nanomechanical devices based mainly on integrated circuit silicon micro- and nanotechnologies (interferometers, plasmonics, microcantilevers, and magnetoplasmonics), microfluidics for biosensing, and surface biofuncionalization by nanometer-scale techniques. The technological platforms developed within the Group are applied in diverse fields such as in environmental control, functional genomics, proteomics, and early diagnostics in medicine. 